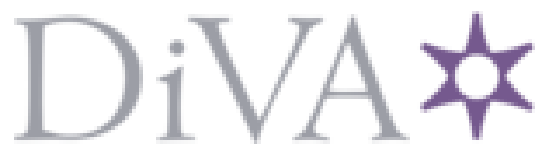

http://www.diva-portal.org

This is the published version of a paper published in ACS Applied Bio Materials.

Citation for the original published paper (version of record):

Tasiopoulos, C P. (2020)

Surface Functionalization of PTFE Membranes Intended for Guided Bone Regeneration

Using Recombinant Spider Silk

ACS Applied Bio Materials, 3(1): 577-583

https://doi.org/10.1021/acsabm.9boo972

Access to the published version may require subscription.

N.B. When citing this work, cite the original published paper.

Permanent link to this version:

http://urn.kb.se/resolve?urn=urn:nbn:se:kth:diva-295256 


\title{
Surface Functionalization of PTFE Membranes Intended for Guided Bone Regeneration Using Recombinant Spider Silk
}

\author{
Christos Panagiotis Tasiopoulos, ${ }^{\dagger}$ Sarunas Petronis, ${ }^{\ddagger}$ Herman Sahlin, ${ }^{\S}$ and My Hedhammar* ${ }^{\dagger}$ (1) \\ ${ }^{\dagger}$ Department of Protein Science, School of Engineering Sciences in Chemistry, Biotechnology and Health, AlbaNova University \\ Center, KTH - Royal Institute of Technology, Stockholm 114 21, Sweden \\ ${ }^{\ddagger}$ Bioscience and Materials, RISE Research Institutes of Sweden, Borås 501 15, Sweden \\ ${ }^{\S}$ Research Department, Neoss Ltd, Göteborg 413 46, Sweden
}

Supporting Information

ABSTRACT: Alveolar bone loss is usually treated with guided bone regeneration, a dental procedure which utilizes a tissue-separation membrane. The barrier membrane prevents pathogens and epithelial cells to invade the bone augmentation site, thereby permitting osteoblasts to deposit minerals and build up bone. This study aims at adding bioactive properties to otherwise inert PTFE membranes in order to enhance cell adherence and promote proliferation. A prewetting by ethanol and stepwise hydration protocol was herein employed to overcome high surface tension of PTFE membranes and allow for a recombinant spider silk protein, functionalized with a cellbinding motif from fibronectin (FN-silk), to self-assemble into a nanofibrillar coating. $\mathrm{HaCaT}$ and U-2 OS cells were seeded onto soft and hard tissue sides, respectively, of membranes coated with FN-silk. The cells could firmly adhere as early as $1 \mathrm{~h}$ post seeding, as well as markedly grow in numbers when kept in culture for 7 days. Fluorescence and scanning electron microscopy images revealed that adherent cells could form a confluent monolayer and develop essential cell-cell contacts during 1

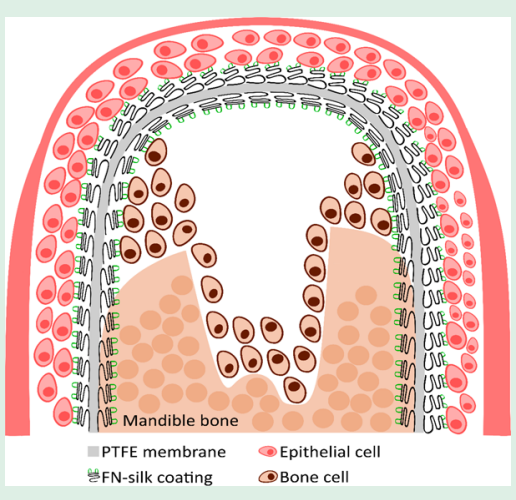
week of culture. Hence, functionalized PTFE membranes have a potential of better integration at the implantation site, with reduced risk of membrane displacement as well as exposure to oral pathogens.

KEYWORDS: guided bone regeneration, nonreinforced PTFE membranes, recombinant spider silk, surface functionalization, cell adherence

\section{INTRODUCTION}

Alveolar bone loss is the condition in which the structural support of a tooth has become impaired due to trauma, disease, or extraction of a decayed tooth. Guided bone regeneration (GBR) is a commonly followed dental procedure when bone loss is evident and full implant osseointegration is needed to allow for functional loading. GBR aims at facilitating bone matrix regeneration around the implant in order to ensure its longevity. It has been reported that the process results in a survival rate of the implant between 79 and $100 \%$ after 5 years of function in bone-regenerated sites. ${ }^{1}$

In GBR, a barrier membrane that prevents epithelial and connective tissue cells from migrating to the defect site is used. Thereby, it permits osteoprogenitor cells to exclusively proliferate and deposit bone content. ${ }^{2,3}$ The first generation of such membranes, still widely used in the clinics today, were nonresorbable and primarily made of inert polymers, such as polytetrafluoroethylene (PTFE). In general, PTFE membranes are stable, biocompatible, and do not elicit immunological responses by the host tissue., ${ }^{4,5}$ Nonetheless, they have been reported to be less prone to support cellular adhesion, ${ }^{3}$ and therefore, the cellular growth and bone restoration may be slower. Furthermore, in some cases, soft tissues retract from the membrane leaving it exposed toward the oral cavity and therefore increasing the risk of infections. Thus, they may often require surface modification in order to enhance cell attachment and improve their osteoinductive properties. ${ }^{6}$ Nevertheless, chemical modification of PTFE membranes is a challenging task because of high inertness and hydrophobicity of the material.

Hydrophobic polymers intended for biomedical applications have previously been successfully coated with recombinant spider silk proteins. ${ }^{7,8}$ The solid hydrophobic substrates allowed the silk proteins to form a coating of various thicknesses and were thereby shown to attain improved wetting capacity. In this study, a strategy for surface functionalization of nonreinforced porous PTFE membranes, specifically used in dentistry, with a functionalized spider silk protein was evaluated. The recombinant spider silk protein 4RepCT, produced by a heterologous bacterial host system, has previously been used as substrate for mammalian cell cultures. ${ }^{9}$ To further add functional properties, the arginine-

Received: October 21, 2019

Accepted: December 23, 2019

Published: December 23, 2019 
glycine-aspartic acid (RGD)-containing cell binding motif found in fibronectin has been coupled at a gene level to 4RepCT. ${ }^{10}$ The functionalized silk protein, denoted FN4RepCT, has been shown to be able to promote cell adhesion and direct growth on a self-assembled nanofibrillar network, ${ }^{11}$ which is similar in morphology to structures in the extracellular matrix (ECM). The nanofibrillar silk coating was shown to increase in thickness as long as silk proteins were available in the bulk. Moreover, the coatings were shown to be stable toward washes with different solutions, such as $\mathrm{NaOH}, \mathrm{EtOH}$, and $\mathrm{HCl}$.

Herein, surfaces of nonreinforced PTFE membranes from Neoss were processed to overcome high surface tension and allow FN-4RepCT to self-assemble into stable nanofibrillar coatings that facilitate cell adherence. The membranes were prewetted by ethanol, hydrated, and coated with FN-4RepCT, and subsequently, the sides intended for interfacing soft and hard tissue were seeded with epithelial- and osteoblast-like cells, respectively. After surface wetting by ethanol, both sides of the membrane were shown to support self-assembly of FN4RepCT into a nanofibrillar coating which augmented cell attachment and sustained cell growth for 7 days.

\section{MATERIALS AND METHODS}

2.1. Cell Cultures. Human keratinocytes $(\mathrm{HaCaT})$ isolated from skin (Cell Lines Service, Heidelberg, Germany) were cultured and expanded in Dulbecco's Modified Eagle Medium/Nutrient Mixture F12 (Gibco, Waltham, MA, U.S.A.) growth medium supplemented with $5 \%$ fetal bovine serum (FBS). U-2 human osteosarcoma cells (U2 OS) isolated from bone (ATCC, Manassas, VA, U.S.A.) were cultured and expanded in McCoy's 5A (modified) (Gibco, Waltham, MA, U.S.A.) growth medium supplemented with $10 \%$ FBS. HaCaT and U-2 OS cells were used at passage 35 and 33, respectively. Growth medium in all cell cultures was changed every second day.

2.2. Preparation of Membranes. PTFE membranes (NeoGen, Nonreinforced membrane, Neoss Ltd., Harrogate, U.K.) were cut using a biopsy punch (Integra, York, PA, U.S.A.) into circular pieces (6 $\mathrm{mm}$ in diameter) to fit in wells of a 96-well plate. The membranes were then submerged in $70 \%$ ethanol, sonicated (Branson 3510, Marshall Scientific, Hampton, NH, U.S.A.) for $15 \mathrm{~min}$ and incubated overnight in $70 \%$ ethanol. The next day, they were serially hydrated in 40 and $20 \%$ ethanol-water solutions for $10 \mathrm{~min}$ each step. The membranes were then submerged in sterile Milli- $Q$ water, sonicated for $10 \mathrm{~min}$, incubated for $10 \mathrm{~min}$ and finally, incubated with sterile phosphate-buffered saline (PBS) for 10 min before coated with the silk protein.

2.3. Coating with FN-Silk. The stock solution of $3.0 \mathrm{mg} / \mathrm{mL}$ of FN-4RepCT protein (from now on denoted FN-silk) in PBS (Spiber Technologies, Stockholm, Sweden) was thawed at room temperature and spun down for a minute using a benchtop centrifuge. The protein was then diluted in PBS to a final concentration of $0.1 \mathrm{mg} / \mathrm{mL}$, spun down for another minute, and finally added to the respective membranes $(n=6)$. After $1 \mathrm{~h}$ of incubation, the protein solution was removed and coated membranes were washed twice with PBS before being seeded with cells.

2.4. Protein Quantification. The FN-silk protein was prepared in solution as described above and the concentration was measured in a spectrophotometer (NanoDrop 1000, Thermo Fisher Scientific, Waltham, MA, U.S.A.) before and after addition to the membranes $(n=8)$. From the measured drop in silk concentration after $1 \mathrm{~h}$ of incubation on the membranes, the amount of silk bound to each membrane $\left(\right.$ per $\mathrm{mm}^{2}$ ) could be obtained.

2.5. Cell Seeding. Cells were harvested when reaching approximately $80 \%$ confluency. Cells were washed once with prewarmed PBS and enzymatically detached with TrypLE Express (Life Technologies, Waltham, MA, U.S.A.), in order to be counted and prepared to $10^{6}$ cells $/ \mathrm{mL}$ solutions. Cell solutions were seeded to result in a final density of $10^{4}$ cells per $0.33 \mathrm{~cm}^{2}$ and $5 \times 10^{3}$ cells per $0.33 \mathrm{~cm}^{2}$ for quick adhesion and expansion analyses, respectively, and incubated for $1 \mathrm{~h}$ at $37^{\circ} \mathrm{C}, 5 \% \mathrm{CO}_{2}$, and $95 \%$ humidity. Nonadherent cells were removed by aspiration, and cells that adhered were further analyzed or kept in culture, as described below.

2.6. Cell Fixation for Quick Adhesion Analysis. To evaluate the degree of adhesion after $1 \mathrm{~h}$ of incubation, adherent cells were washed twice with prewarmed PBS, and fixed either with $4 \%$ paraformaldehyde (PFA) in PBS for immunostaining or $2 \%$ glutaraldehyde (Sigma-Aldrich, St. Louis, MO, U.S.A.) in $0.1 \mathrm{M}$ HEPES buffer for scanning electron microscopy (SEM).

2.7. Immunostainings. After fixation with 4\% PFA, cells were permeabilized with $0.2 \%$ Triton X-100 in PBS, washed twice with $0.05 \%$ Tween in PBS for 5 min, and finally, blocked with $1 \%$ bovine serum albumin (BSA) in PBS for 60 min. Alexa Fluor 488 Phalloidin (Thermo Fisher Scientific, Waltham, MA, U.S.A.) 1:40 in 1\% BSA in PBS was used to stain the actin filaments for $2 \mathrm{~h}$ at room temperature, before nuclear staining with DAPI for $10 \mathrm{~min}$. Stained cells were finally washed twice with $0.05 \%$ Tween in PBS for 5 min, mounted on microscopic glasses using Dako fluorescence mounting medium (Dako North America, Carpinteria, CA, U.S.A.), and documented using an inverted fluorescence microscope (Nikon Eclipse Ti, Tokyo, Japan). Images at $10 \times$ magnification were captured using the NIS elements BR software.

2.8. SEM-EDS Analysis. PTFE membranes seeded with cells were chemically fixed and dehydrated prior to the analysis. Primary fixation was done by $2 \%$ glutaraldehyde, after which fixed samples were kept and transported at $+4{ }^{\circ} \mathrm{C}$ until secondary fixation with $1 \%$ osmium tetroxide (Sigma-Aldrich, St. Louis, MO, U.S.A.) for $1 \mathrm{~h}$. The samples were then rinsed twice with deionized water for $10 \mathrm{~min}$, serially dehydrated in $30,50,75,90$, and $100 \%$ ethanol in deionized water for $10 \mathrm{~min}$ each step, and finally treated by 50 and $100 \%$ hexamethyldisilazane (Sigma-Aldrich, St. Louis, MO, U.S.A.) in 100\% ethanol for $10 \mathrm{~min}$ each step. The samples were then let to dry overnight at room temperature in a desiccator. The next day, fixed samples were mounted on microscopy pins with adhesive carbon pads (Agar Scientific, Stansted, U.K.) and coated with $10 \mathrm{~nm}$ thick $\mathrm{Au} / \mathrm{Pd}$ conductive film (Gatan Inc., Pleasanton, CA, U.S.A.). Adherent cells on PTFE membranes were imaged with a Zeiss SUPRA 40VP scanning electron microscope operated in secondary electron mode at $4.05 \mathrm{kV}$ acceleration voltage, 3-15 mm working distance, and 100$200000 \times$ magnification range. PTFE membranes without cells were prepared and imaged in the same manner excluding chemical fixation and dehydration steps.

$\mathrm{X}$-ray energy dispersion spectroscopy (EDS) was performed using X-Max $20 \mathrm{~mm}^{2}$ EDS detector (Oxford Instruments, Abingdon, U.K.) mounted on the same SEM instrument and INCA 4.15 Microanalysis Suite software (Oxford Instruments Analytical). Elemental composition of bare $(n=2)$ and FN-silk coated $(n=5)$ PTFE membranes was analyzed. $\mathrm{Au}$ and $\mathrm{Pd}$ elements were excluded from the chemical composition evaluation as they represented anticharging film coating, introduced during sample preparation.

2.9. Cell Viability and Proliferation Assay. Viable and proliferative cells were evaluated with alamarBlue viability assay (Invitrogen, Waltham, MA, U.S.A.). After the initial $1 \mathrm{~h}$ of incubation, cells were washed twice with prewarmed PBS before addition of growth medium and further cell culture. Cell growth was monitored at days $1,3,5$, and 7 after seeding by addition of alamarBlue diluted 1:10 in respective growth media, followed by $2 \mathrm{~h}$ of incubation. Wells $(n=$ 4) without cells were used as blank. Fluorescence intensity of $100 \mu \mathrm{L}$ of supernatants was read out in a fluorescence plate reader (CLARIOstar, BMG Labtech, Ortenberg, Germany) using excitation at $544 \mathrm{~nm}$ and emission at $595 \mathrm{~nm}$.

2.10. Static Water Contact Angle Analysis. Water contact angles were measured in a static mode by a goniometer (DSA100, Krüss, Hamburg, Germany) using 2-3 $\mu \mathrm{L}$ sessile water droplets. Sessile droplets $(n=6)$ were analyzed for each sample using Krüss DSA software and Young-Laplace fitting of droplet shape. The measurements were carried out in a temperature- and humiditycontrolled laboratory. 
2.11. Infrared Spectroscopy. Nicolet 6700 FT-IR (Thermo Electron Corporation, Madison, WI, U.S.A.) equipped with a single bounce attenuated total reflectance diamond insert (Smart Orbit) was used for the IR characterizations. Thirty-two scans with $4 \mathrm{~cm}^{-1}$ resolution were averaged from each sample and were referenced against ambient air by using OMNIC software suite. The referenced spectra were further exported to CSV format in order to be leveled, normalized, and plotted using MS Excel software.

2.12. Statistics. Multiple $t$ tests followed by Holm-Sidak method were performed using GraphPad Prism version 6.05 for Windows (GraphPad Software, La Jolla, CA, U.S.A.). Statistical significance was considered as $* p<0.05, * * p<0.01$, and $* * * p<0.001$.

\section{RESULTS}

Nonreinforced PTFE membranes from Neoss have a two-layer design with different surface roughness and porosity (Figure S1), which enables them to be stable and mediates osseointegration in the bone augmentation site. In particular, the side intended for hard tissue features an expanded surface texture which induces osteogenesis. The opposite side favors soft tissue interaction and acts as a barrier to bacteria, thereby minimizing the risk of infection. In the first trials to coat surfaces by incubation in the silk protein, the hydrophobic nature of the membrane did not allow a water-based protein solution to come in close enough contact for the silk to selfassemble into a nanofibrillar coating. Therefore, a mild surface treatment protocol (Figure 1) was established in order to

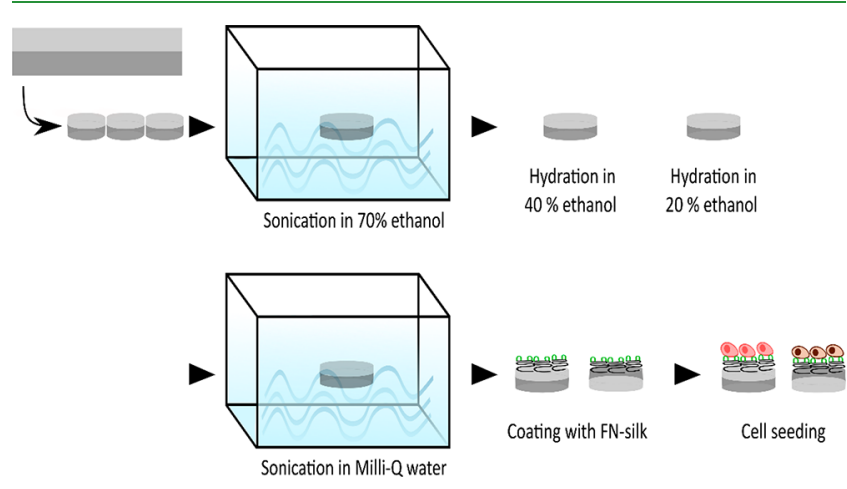

Figure 1. Established protocol for surface treatment of PTFE membranes.

prewet both soft and hard tissue sides of the PTFE membrane. The protocol involved sonication-assisted wetting by ethanol and serial hydration steps with different concentrations of ethanol-water solutions. Ethanol has a low surface tension and can be used for the wetting of otherwise hydrophobic substrates. ${ }^{12,13}$ Because ethanol is antiseptic and widely used in healthcare, it was considered to be optimal for treatment of the membranes prior to coating with the silk protein.

In the absence of surface prewetting, FN-silk formed a sheetlike superficial coating which completely masks the delicate textures of the membrane (Figure S2). The pores of the membrane were completely clogged by the protein, and therefore, membrane morphological and transport properties required for a successful GBR are affected. On the contrary, prewetting of the substrate by ethanol permitted complete access to the water-based silk protein solution to the pores and internal network of the PTFE membrane and enabled the protein to self-assemble into a nanofibrillar coating (Figure 2). Instead of forming a pore-bridging sheet, the silk fibrillated as a porous network between the nodes and fibrils that feature the

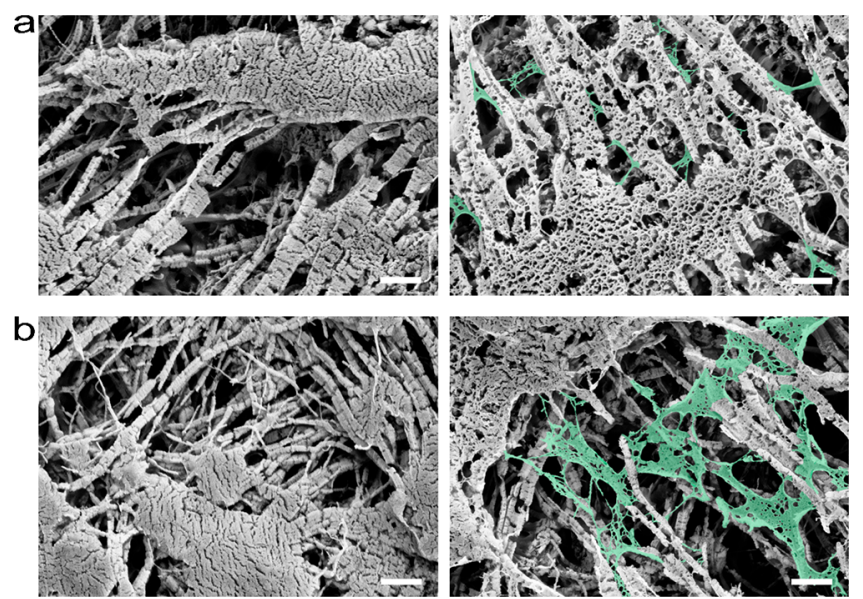

Figure 2. SEM images of PTFE membranes. (a) Micrographs of noncoated (left) and FN-silk coated (right) soft tissue sides. (b) Micrographs of noncoated (left) and FN-silk coated (right) hard tissue sides. Example of false colored green silk nanofibrils on FN-silk coated soft and hard tissue sides of PTFE membranes. Scale bars $=1$ $\mu \mathrm{m}$.

matrix of such membranes and, most importantly, did not clog the pores. The silk nanofibrils were observed to be of various thicknesses, but all are within the nanoscale range (Figure S3). Based on the measured reduction of silk concentration in solution after $1 \mathrm{~h}$ of incubation on the substrates, the amount of silk bound to each membrane was estimated to be $1.77 \times$ $10^{-4} \mathrm{mg} / \mathrm{mm}^{2}$. The presence of silk on the coated membranes could also be confirmed by the difference in elemental composition detected from SEM-EDS analysis (Table S1). Furthermore, the uniform distribution of silk nanofibrils is corroborated by the characteristic amine peaks, as compared with $\mathrm{C}-\mathrm{F}$ peak intensities from bare PTFE substrates, detected on different membrane locations in FTIR analysis (Figure S4).

To evaluate the degree of surface wettability, noncoated as well as FN-silk coated soft and hard tissue sides of the membranes were subjected to static water contact angle analysis. Unprocessed and noncoated soft and hard tissue sides of the membranes exhibited high contact angles (143.9 and 152 degrees on average, respectively), which hints at weak solid-liquid interaction strength. On the contrary, both tissue sides were found to have improved the degree of wetting once processed and coated with FN-silk (106 and 105 degrees on average, respectively) (Figure S5).

Once the silk self-assembled into a nanofibrillar coating onto the PTFE surface, the degree of cell adherence on coated as well as noncoated membranes was studied. The functionalized silk protein, with the RGD-containing cell binding motif from fibronectin, was herein shown to enhance cell adherence. Fluorescence images of cells stained for actin filaments (Figure $3 \mathrm{a}, \mathrm{b})$ revealed a remarkable increase in the number of adherent cells on the surfaces coated with FN-silk, as compared to noncoated controls. In particular, cells seeded onto the substrates coated with the silk protein seemed to have established firm adhesion and acquired a spread-out morphology. Adherent cells attain a flattened morphology, as an indication of sound compliance with the substrate, as early as 30 min post seeding. ${ }^{14}$ To further shed light on the above observations, SEM analysis was performed on fixed cells after adherence for $1 \mathrm{~h}$ onto surfaces coated with FN-silk, as well as to noncoated controls. As expected, acquired images revealed 

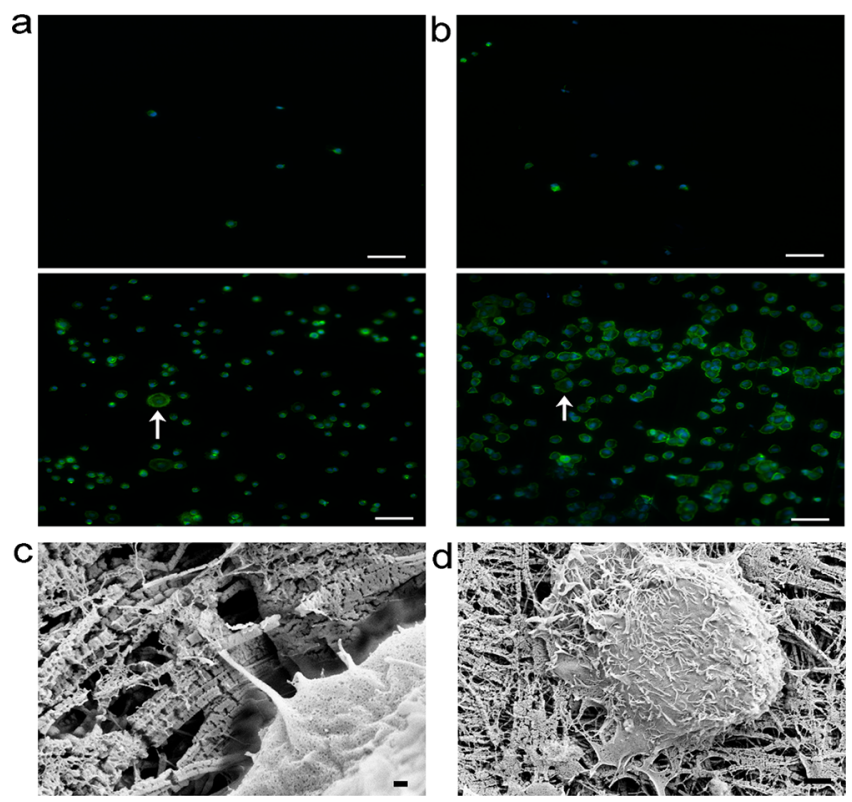

Figure 3. Fluorescence and SEM images of fixed cells after $1 \mathrm{~h}$ post seeding. (a) Micrographs (10X) of HaCaT cells stained for F-actin (green) and DAPI (blue) seeded on noncoated (upper left panel) and coated with FN-silk (middle left panel) soft tissue sides. (b) Micrographs $(10 \times)$ of U-2 OS cells stained for F-actin (green) and DAPI (blue) seeded on noncoated (upper right panel) and coated with FN-silk (middle right panel) hard tissue sides. White arrows point to example of an adherent cell on soft and hard tissue sides, respectively. Scale bars $=100 \mu \mathrm{m}$. (c) Micrograph of a HaCaT cell on soft tissue side coated with FN-silk (lower left panel). Scale bar $=200$ nm. (d) Micrograph of a U-2 OS cell on hard tissue side coated with FN-silk (lower right panel). Scale bar $=2 \mu \mathrm{m}$. Seeding density: 10000 cells per $100 \mu \mathrm{L}$.

cytoplasmic outgrowths (Figure 3c,d) developed by both $\mathrm{HaCaT}$ and U-2 OS cells seeded onto the FN-silk coated soft and hard tissue sides, respectively.

The proliferation rate of adherent cells was further evaluated every second day with alamarBlue cell viability assay. The coating of FN-silk was herein shown to sustain a remarkable growth of cells on both sides of the PTFE membrane, as compared with noncoated controls (Figure 4). Both soft and hard tissue sides treated with the established surface treatment protocol but not coated with the protein were unable to promote cell proliferation because of poor attachment at a

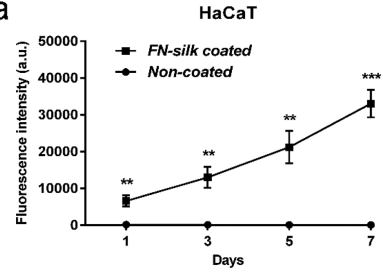

b

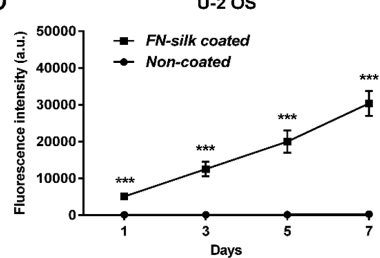

Figure 4. Growth rate and viability of $\mathrm{HaCaT}$ and U-2 OS cells in culture for 7 days. (a) alamarBlue cell viability assay (mean \pm SEM, $n$ $=6$ ) of $\mathrm{HaCaT}$ cells seeded on noncoated (full circle) and coated with FN-silk (full square) soft tissue sides of PTFE membranes. (b) alamarBlue cell viability assay (mean \pm SEM, $n=6$ ) of U-2 OS cells seeded on noncoated (full circle) and coated with FN-silk (full square) hard tissue sides of PTFE membranes. Seeding density: 5000 cells per $100 \mu \mathrm{L}$. Statistics: $* p<0.05, * * p<0.01$, and $* * * p<0.001$ (multiple $t$ tests). seeding. On the contrary, $\mathrm{HaCaT}$ and U-2 OS cells were found to markedly grow in numbers when seeded to the respective sides that were coated with the silk protein. The growth curves revealed a linearly increasing metabolic activity, which hints that cells seeded onto coated surfaces were highly viable and active up to 7 days in culture. The observed growth can be explained by the firm initial adherence mediated by cellsubstrate binding and the fact that seeded cells were close enough to establish cell-cell contacts.

Lastly, the ability of cells to reach confluency on PTFE membranes was examined. Confluent cell layers were found covering the entire FN-silk coated soft and hard tissue sides of the membrane (Figure S6). This observation indicates that the FN-silk coatings were sufficiently complete and uniform across the entire membrane surface area. Further, fluorescence images revealed that cells were in close proximity to interact and form a cell monolayer (Figure 5a,b). In particular, $\mathrm{HaCaT}$ cells were
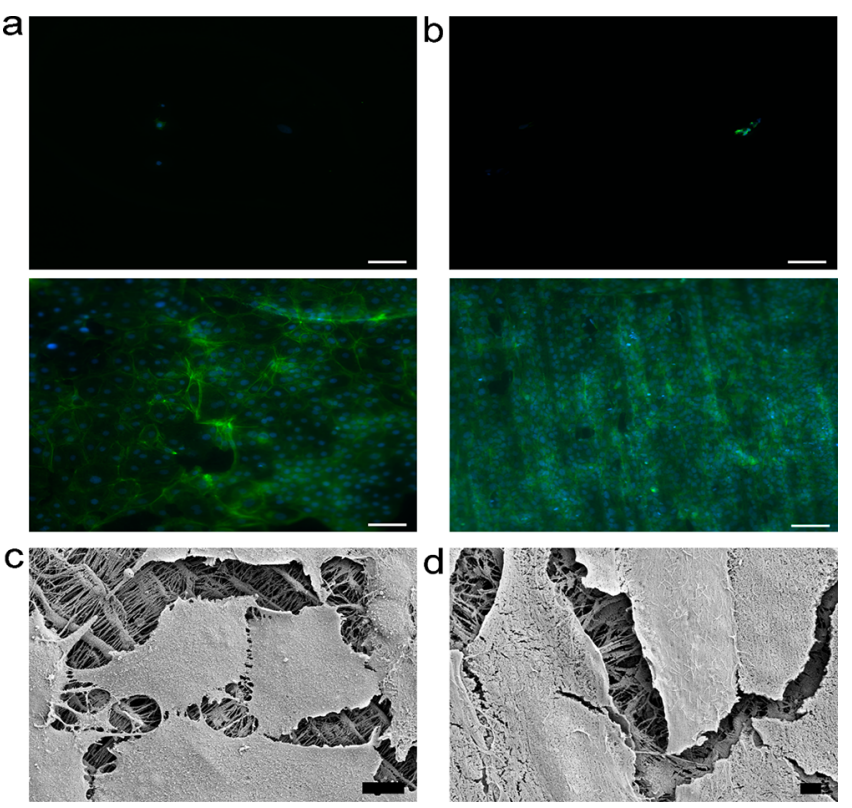

Figure 5. Fluorescence and SEM images of fixed cells after 7 days in culture. (a) Micrographs (10X) of $\mathrm{HaCaT}$ cells stained for F-actin (green) and DAPI (blue) seeded on noncoated (upper left panel) and coated with FN-silk (middle left panel) soft tissue sides. (b) Micrographs (10X) of U-2 OS cells stained for F-actin (green) and DAPI (blue) seeded on noncoated (upper right panel) and coated with FN-silk (middle right panel) hard tissue sides. Scale bars $=100$ $\mu \mathrm{m}$. (c) Micrograph of $\mathrm{HaCaT}$ cells on soft tissue side coated with FN-silk (lower left panel). Scale bar $=10 \mu \mathrm{m}$. (d) Micrograph of U-2 OS cells on hard tissue side coated with FN-silk (lower right panel). Scale bar $=3 \mu \mathrm{m}$. Seeding density: 5000 cells per $100 \mu \mathrm{L}$.

found to have uniformly spread over the entire FN-silk coated surface with flattened morphology of cell nuclei. It has been suggested that a more rigid substrate results in a flattened cell nucleus, which is an indication of better cell spreading. ${ }^{15}$ In addition, surface wettability may influence protein adsorption and consequently cell spreading; along with surface roughness. ${ }^{16}$ U-2 OS cells on hard tissue sides coated with FN-silk were also found to have clustered and resulted in a confluent monolayer. To summarize, the silk coated surfaces were herein shown to allow for both $\mathrm{HaCaT}$ and U-2 OS cells to become confluent and establish cell-cell contacts after 7 days in culture (Figure 5c,d). 


\section{DISCUSSION}

The PTFE is a hydrophobic material described by low surface energy and high contact angle (>110 degrees) to water-based compounds. ${ }^{17}$ The water contact angle further increases due to the porosity of extended PTFE and can reach up to 160 degrees. ${ }^{18}$ Several strategies have been employed aiming at improving the wetting capacity of hydrophobic materials and thereby protein adsorption (e.g., bombardment with ions, irradiation with ultraviolet light, and plasma discharge). ${ }^{19}$ Except for lowering surface tension, the aforementioned treatment methods have been acknowledged for enhancing in vitro cell adhesion as well. ${ }^{20-22}$ Harsh as well as milder surfactants have also been used to improve the wetting and make PTFE substrates more favorable to protein-based solutions. ${ }^{23,24}$ Recently, the addition of ethanol to water has been proposed as means to decrease capillary interactions and contact angle to a PTFE-like substrate. ${ }^{12}$

In order to achieve a complete wetting of the porous PTFE membrane, a liquid that intrinsically has a low contact angle ( $<90 \mathrm{deg}$ ) on the material has to be utilized and then gradually being replaced by water, without introducing entrapped air. The lowest contact angle toward PTFE is reported to be possessed by nonpolar aprotic liquids (e.g., heptane, octane, decane, tridecane, etc) ${ }^{25}$ However, miscibility with water, a common property of polar solvents, such as acetone, ethanol, $n$-propanol, and methanol, is also important at selecting a proper prewetting agent. The contact angle of these solvents has also been reported to be sufficiently low (40-50 degrees), and thus, they can facilitate the wetting of PTFE substrates. ${ }^{26}$ Further, it is also of importance to consider the environmental, health, and safety (EHS) properties of the prewetting medium, especially when the intended use is in the medical field. Based on a comparison study of the most commonly used solvents, ${ }^{27}$ ethanol raises the least EHS concerns and therefore was chosen to prewet the PTFE membranes in this study. Herein, the silk protein was shown able to self-assemble into a nanofibrillar network (Figure 2) to the PTFE membranes, which were wetted by ethanol and serially hydrated. In contrast, a thick superficial sheet-like coating (Figure S2) was formed to nontreated control membranes, in which the pores were found completely clogged by the silk protein. It is speculated that this occurred because of the porous structure and poor wettability of the unmodified PTFE material, causing FN-silk to form a sheet at the interface between entrapped air within the membrane and the surrounding liquid media.

It has previously been reported that silk proteins adsorb at a constantly increasing rate on a surface and, through proteinprotein interactions, form patterns similar to the ECM within connective tissues. ${ }^{11}$ Thereby, added cells can sense a microenvironment similar to the native and develop essential cell-ECM attachment points through cell receptors. ${ }^{10}$ Many of these cell receptors are transmembrane proteins (e.g., integrins) that bind to ECM proteins (e.g., fibronectin and laminin) and key mediators in cell adherence, migration, and proliferation. In this study, cell adherence was found to have markedly been enhanced when $\mathrm{HaCaT}$ and U-2 OS cells were seeded to the respective soft and hard tissue sides of membranes coated with FN-silk (Figure 3a,b). Further, cells displayed characteristic cytoplasmic outgrowths (Figure $3 c, d$ ). The cellular fate is particularly dictated by the ability of a cell to generate integrin-ligand binding sites with the underlying substrate. The amount of those generated sites may sustain viability and promote growth or lead to programmed cell death. As indicated from the proliferation data, cell growth could be promoted by the FN-silk coated membranes, whereas cells seeded to noncoated controls were unable to remain viable and proliferate (Figure 4). Further, surface polarity seems to also have an impact on the development of integrin binding by anchorage-dependent cells. For example, cells may experience difficulties in recognizing adhesion molecules (e.g., RGD) on too hydrophobic surfaces, whereas extremely hydrophilic ones result in a rounded cell morphology which ultimately leads to apoptosis. ${ }^{19}$

The RGD peptide is well recognized as a bioactive motif that enhances in vitro cell attachment via integrins. ${ }^{28}$ Thus, it is often used to add functionality and improve surface properties of inert biomaterials. ${ }^{29}$ A silk variant functionalized with the RGD-containing cell binding motif from fibronectin was herein shown to have indeed added the essential bioactive properties to PTFE membranes. Integrin-mediated adhesion is also promoting cell proliferation provided by the native or an ECM-like environment. ${ }^{30}$ In particular, adherent cells are able to sense the stiffness of the ECM and accordingly direct motility and migration. Recently, it has also been reported that the rigidity of a substrate influences cytoskeletal rearrangement in polarized cells, ultimately affecting cellular growth. ${ }^{31}$ Hence, a material able to both mechanically and biologically modify a polymeric surface in order to promote cellular growth is important in tissue integration applications. The 4RepCT silk protein fused with cell adhesion motifs drastically increased proliferation of the various cell lines studied previously. ${ }^{10,32}$ In addition, its exceptional mechanical properties render it an optimal candidate to be used as a coating to polymeric substrates. $^{33}$

Cell flattening is often attributed to epithelial cells lining organs and inner surfaces throughout the human body with the primary function to act as a barrier, thereby protecting underlying tissues from invasive pathogens. Further, flattened cell morphology is also an indication of cell-matrix compliance mainly regulated by substrate rigidity. ${ }^{34,35}$ Cell-cell junctions in epithelial cells mediate cell communication and polarization $^{36}$ and maintains tissue integrity and homeostasis. ${ }^{37,38}$ In addition, junctional complexes provide mechanical support to cells and tissues in order to protect them from abrasion. In this study, both examined cell types were shown to have flattened out and developed cell-cell contacts at the last day of culture (Figure 5c,d). Cell-cell interaction is also essential in bone remodelling where osteoblasts should be found in close proximity, in order to transduce biological signals into bone formation. ${ }^{39}$ Moreover, the spatial-temporal interaction between bone-forming and bone-resorptive cells have also been lately found to regulate bone homeostasis. ${ }^{40}$

The FN-silk protein was herein shown to provide both tissue sides of the PTFE membrane with the necessary bioactive features that can lead to an efficient replication of the matrix around lost alveolar bone. It has been reported that organic matrix deposition and bone mineralization is initiated after osteoblasts have become confluent and formed a monolayer in two-dimensional cultures. ${ }^{41}$ It is nonetheless of importance to investigate whether seeded U-2 OS cells onto silk-coated hard tissue sides have started the deposition of minerals. Bone mineralization is a process mainly mediated by a collagen template, in which mineral crystals are deposited in a hierarchically organized fashion around the collagen fibrils. Further studies are hence needed to probe U-2 OS cells for 
collagen as well as minerals deposition onto the silk-coated hard tissue side.

In addition, recombinant spider silk can be conjugated with enzymes that target bacteria ${ }^{42}$ or fused with antibiotics. ${ }^{43}$ The strategies aim at lysing bacteria once bound to a surface or not permitting them to form a biofilm which is necessary for their survival. The approach has mainly been regarded for dental implants and therefore, FN-silk coated soft tissue sides of PTFE membranes can be exposed to certain pathogens found in the oral cavity. The answer to whether microbes can become less adherent or lysed once bound to silk-coated soft tissue side may pave the way to consider the material for further in vivo studies.

\section{CONCLUSIONS}

Alveolar bone loss requires guided bone regeneration to ensure dental implant's stability and longevity. Nonetheless, matrix remodelling may be too time-consuming, and therefore, novel techniques with the ability to reduce the overall time needed for induced osteogenesis have to be employed. Further, it is also important to maintain an intact soft tissue coverage over the tissue-separation membrane in order to prevent infections. Herein, a well-studied material commonly used in GBR procedures was coated with the FN-silk protein, which aided cell adherence and proliferation. A surface treatment protocol established to prewet PTFE membranes allowed the silk protein to self-assemble into a nanofibrillar coating, which is similar in morphology to ECM structures. Seeded cells could immediately recognize the ECM-like microenvironment and develop firm attachment through cell-substrate binding. Over a period of 7 days in culture, adherent cells could markedly grow in numbers, become confluent, and form essential cellcell contacts on the silk-coated membranes. In contrast, no significant cell attachment and proliferation could be observed on the noncoated control membranes. Thus, a reliable and simple technique has been established that can add bioactive properties to an inert material and further be considered for in vivo studies.

\section{ASSOCIATED CONTENT}

\section{(S Supporting Information}

The Supporting Information is available free of charge at https://pubs.acs.org/doi/10.1021/acsabm.9b00972.

SEM images of PTFE membranes, nontreated hard tissue side of PTFE membrane coated with FN-silk, and silk nanofibrils on FN-silk coated soft and hard tissue sides. Elemental composition analysis performed by SEM - EDS and FTIR analysis on noncoated and FNsilk coated PTFE membranes. Static water contact angle analysis on PTFE membranes, and fluorescence images on fixed cells on noncoated as well as FN-silk coated soft and hard tissue sides after 7 days in culture (PDF)

\section{AUTHOR INFORMATION}

\section{Corresponding Author}

*E-mail: myh@kth.se.

\section{ORCID $\odot$}

My Hedhammar: 0000-0003-0140-419X

\section{Author Contributions}

M.H., S.P., and H.S. conceived and directed the research. M.H. and C.P.T. designed the experiments. C.P.T. performed cell experiments and analysis. C.P.T. and S.P. designed and conducted scanning electron microscopy analysis. C.P.T. wrote the manuscript. All authors discussed the results, commented on the manuscript, and have given approval to the final version of it.

\section{Funding}

This research was supported by Vinnova, Swedish Research Council and Knut \& Alice Wallenberg foundation.

\section{Notes}

The authors declare the following competing financial interest(s): M.H. has shares in Spiber Technologies $A B$, a company that aims to commercialize recombinant spider silk.

\section{ACKNOWLEDGMENTS}

We thank Spiber Technologies AB for generously providing the silk protein used in all experiments of this study, as well as Neoss Limited for kindly providing the nonreinforced PTFE membranes to work with.

\section{ABBREVIATIONS}

GBR, guided bone regeneration PTFE, polytetrafluoroethylene

RGD, arginine-glycine-aspartic acid

ECM, extracellular matrix

$\mathrm{HaCaT}$, human keratinocytes

FBS, fetal bovine serum

U-2 OS, human osteosarcoma cells

PBS, phosphate-buffered saline

FN-silk, FN-4RepCT

PFA, paraformaldehyde

SEM, scanning electron microscopy

BSA, bovine serum albumin

EDS, energy dispersion spectroscopy

EHS, environmental health and safety

\section{REFERENCES}

(1) Hammerle, C. H.; Jung, R. E.; Feloutzis, A. A systematic review of the survival of implants in bone sites augmented with barrier membranes (guided bone regeneration) in partially edentulous patients. J. Clin. Periodontol. 2002, 29 (s3), 226-231 discussion 232-33..

(2) Retzepi, M.; Donos, N. Guided Bone Regeneration: biological principle and therapeutic applications. Clin Oral Implants Res. 2010, 21 (6), 567-76.

(3) Dimitriou, R.; Mataliotakis, G. I.; Calori, G. M.; Giannoudis, P. $V$. The role of barrier membranes for guided bone regeneration and restoration of large bone defects: current experimental and clinical evidence. BMC Med. 2012, 10, 81.

(4) Liu, J.; Kerns, D. G. Mechanisms of guided bone regeneration: a review. Open Dent. J. 2014, 8, 56-65.

(5) Elgali, I.; Omar, O.; Dahlin, C.; Thomsen, P. Guided bone regeneration: materials and biological mechanisms revisited. Eur. J. Oral Sci. 2017, 125 (5), 315-337.

(6) Gentile, P.; Chiono, V.; Tonda-Turo, C.; Ferreira, A. M.; Ciardelli, G. Polymeric membranes for guided bone regeneration. Biotechnol. J. 2011, 6 (10), 1187-97.

(7) Wohlrab, S.; Spiess, K.; Scheibel, T. Varying surface hydrophobicities of coatings made of recombinant spider silk proteins. $J$. Mater. Chem. 2012, 22 (41), 22050-22054.

(8) Harris, T. I.; Gaztambide, D. A.; Day, B. A.; Brock, C. L.; Ruben, A. L.; Jones, J. A.; Lewis, R. V. Sticky Situation: An Investigation of Robust Aqueous-Based Recombinant Spider Silk Protein Coatings and Adhesives. Biomacromolecules 2016, 17 (11), 3761-3772. 
(9) Widhe, M.; Bysell, H.; Nystedt, S.; Schenning, I.; Malmsten, M.; Johansson, J.; Rising, A.; Hedhammar, M. Recombinant spider silk as matrices for cell culture. Biomaterials 2010, 31 (36), 9575-85.

(10) Widhe, M.; Shalaly, N. D.; Hedhammar, M. A fibronectin mimetic motif improves integrin mediated cell biding to recombinant spider silk matrices. Biomaterials 2016, 74, 256-66.

(11) Nileback, L.; Hedin, J.; Widhe, M.; Floderus, L. S.; Krona, A.; Bysell, H.; Hedhammar, M. Self-Assembly of Recombinant Silk as a Strategy for Chemical-Free Formation of Bioactive Coatings: A RealTime Study. Biomacromolecules 2017, 18 (3), 846-854.

(12) Fritzsche, J.; Peuker, U. A. Wetting and adhesive forces on rough surfaces - An experimental and theoretical study. New Paradigm of Particle Science and Technology. Procedia Eng. 2015, $102,45-53$.

(13) Lundgren, M.; Allan, N. L.; Cosgrove, T.; George, N. Wetting of water and water/ethanol droplets on a non-polar surface: A molecular dynamics study. Langmuir 2002, 18 (26), 10462-10466.

(14) Koller, M. R.; Papoutsakis, E. T. Effect of Cell Adhesion on Cellular Processes and Growth. In Cell adhesion: fundamentals and biotechnological applications; Hjortso, M. A., Roos, J. W., Eds.; Marcel Dekker, Inc.: New York, U.S.A., 1995; Vol. 80, pp 76-78.

(15) Neelam, S.; Hayes, P. R.; Zhang, Q.; Dickinson, R. B.; Lele, T. $\mathrm{P}$. Vertical uniformity of cells and nuclei in epithelial monolayers. Sci. Rep. 2016, 6, 19689.

(16) Sammons, R. L.; Lumbikanonda, N.; Gross, M.; Cantzler, P. Comparison of osteoblast spreading on microstructured dental implant surfaces and cell behavior in an explant model of osseointegration. A scanning electron microscopic study. Clin Oral Implants Res. 2005, 16 (6), 657-66.

(17) Zdziennicka, A. The wettability of polytetrafluoroethylene and polymethylmethacrylate by aqueous solutions of Triton X-100 and propanol mixtures. Appl. Surf. Sci. 2009, 255 (6), 3801-3810.

(18) Zhang, J.; Li, J. A.; Han, Y. C. Superhydrophobic PTFE Surfaces by Extension. Macromol. Rapid Commun. 2004, 25 (11), $1105-1108$

(19) Bacakova, L.; Filova, E.; Rypacek, F.; Svorcik, V.; Stary, V. Cell adhesion on artificial materials for tissue engineering. Physiol Res. 2004, 53 (Suppl1), S35-S45.

(20) Pignataro, B.; Conte, E.; Scandurra, A.; Marletta, G. Improved cell adhesion to ion beam-irradiated polymer surfaces. Biomaterials 1997, 18 (22), 1461-70.

(21) Garcia-Contreras, R.; Kanagawa, S.; Beppu, Y.; Nagao, T.; Sakagami, H.; Nakajima, H.; Shimada, J.; Adachi, K. Morphological features of osteoblasts cultured on ultraviolet-irradiated titanium plates. In Vivo 2011, 25 (4), 649-655.

(22) Lerman, M. J.; Lembong, J.; Muramoto, S.; Gillen, G.; Fisher, J. P. The Evolution of Polystyrene as a Cell Culture Material. Tissue Eng., Part B 2018, 24 (5), 359-372.

(23) Chaudhuri, R. G.; Sunayana, S.; Paria, S. Wettability of a PTFE surface by cationic-non-ionic surfactant mixtures in the presence of electrolytes. Soft Matter 2012, 8 (20), 5429-5433.

(24) Szymczyk, K.; Janczuk, B. Wettability of a polytetrafluoroethylene surface by an aqueous solution of two nonionic surfactant mixtures. Langmuir 2007, 23 (17), 8740-8746.

(25) Lee, S.; Park, J. S.; Lee, T. R. The Wettability of Fluoropolymer Surfaces: Influence of Surface Dipoles. Langmuir 2008, 24 (9), 48174826.

(26) Busscher, H. J.; Arends, J. Determination of the Surface Forces Gamma-S-D and Gamma-S-P from Contact-Angle Measurements on Polymers and Dental Enamel. J. Colloid Interface Sci. 1981, 81 (1), $75-79$.

(27) Capello, C.; Fischer, U.; Hungerbuhler, K. What is a green solvent? A comprehensive framework for the environmental assessment of solvents. Green Chem. 2007, 9 (9), 927-934.

(28) Van Agthoven, J. F.; Xiong, J. P.; Alonso, J. L.; Rui, X.; Adair, B. D.; Goodman, S. L.; Arnaout, M. A. Structural basis for pure antagonism of integrin alphaVbeta3 by a high-affinity form of fibronectin. Nat. Struct. Mol. Biol. 2014, 21 (4), 383-8.
(29) Bellis, S. L. Advantages of RGD peptides for directing cell association with biomaterials. Biomaterials 2011, 32 (18), 4205-10.

(30) Schwartz, M. A.; Assoian, R. K. Integrins and cell proliferation: regulation of cyclin-dependent kinases via cytoplasmic signaling pathways. J. Cell Sci. 2001, 114 (Pt 14), 2553-2560.

(31) Kim, Y.; Ko, H.; Kwon, I. K.; Shin, K. Extracellular Matrix Revisited: Roles in Tissue Engineering. Int. Neurourol J. 2016, 20 (Suppl 1), S23-29.

(32) Widhe, M.; Johansson, U.; Hillerdahl, C. O.; Hedhammar, M. Recombinant spider silk with cell binding motifs for specific adherence of cells. Biomaterials 2013, 34 (33), 8223-34.

(33) Bowen, C. H.; Dai, B.; Sargent, C. J.; Bai, W.; Ladiwala, P.; Feng, H.; Huang, W.; Kaplan, D. L.; Galazka, J. M.; Zhang, F. Recombinant Spidroins Fully Replicate Primary Mechanical Properties of Natural Spider Silk. Biomacromolecules 2018, 19 (9), 38533860.

(34) Eisenberg, J. L.; Safi, A.; Wei, X.; Espinosa, H. D.; Budinger, G. S.; Takawira, D.; Hopkinson, S. B.; Jones, J. C. Substrate stiffness regulates extracellular matrix deposition by alveolar epithelial cells. Res. Rep. Biol. 2011, 2011 (2), 1-12.

(35) Nisenholz, N.; Rajendran, K.; Dang, Q.; Chen, H.; Kemkemer, R.; Krishnan, R.; Zemel, A. Active mechanics and dynamics of cell spreading on elastic substrates. Soft Matter 2014, 10 (37), 7234-46.

(36) Giepmans, B. N.; van Ijzendoorn, S. C. Epithelial cell-cell junctions and plasma membrane domains. Biochim. Biophys. Acta, Biomembr. 2009, 1788 (4), 820-31.

(37) Cavey, M.; Lecuit, T. Molecular bases of cell-cell junctions stability and dynamics. Cold Spring Harbor Perspect. Biol. 2009, 1 (5), a002998.

(38) Garcia, M. A.; Nelson, W. J.; Chavez, N. Cell-Cell Junctions Organize Structural and Signaling Networks. Cold Spring Harbor Perspect. Biol. 2018, 10 (4), a029181.

(39) Civitelli, R. Cell-cell communication in the osteoblast/ osteocyte lineage. Arch. Biochem. Biophys. 2008, 473 (2), 188-92.

(40) Furuya, M.; Kikuta, J.; Fujimori, S.; Seno, S.; Maeda, H.; Shirazaki, M.; Uenaka, M.; Mizuno, H.; Iwamoto, Y.; Morimoto, A.; Hashimoto, K.; Ito, T.; Isogai, Y.; Kashii, M.; Kaito, T.; Ohba, S.; Chung, U. I.; Lichtler, A. C.; Kikuchi, K.; Matsuda, H.; Yoshikawa, H.; Ishii, M. Direct cell-cell contact between mature osteoblasts and osteoclasts dynamically controls their functions in vivo. Nat. Commun. 2018, 9 (1), 300

(41) Taylor, S. E.; Shah, M.; Orriss, I. R. Generation of rodent and human osteoblasts. BoneKEy Rep. 2014, 3, 585.

(42) Nileback, L.; Widhe, M.; Seijsing, J.; Bysell, H.; Sharma, P. K.; Hedhammar, M. Bioactive Silk Coatings Reduce the Adhesion of Staphylococcus aureus while Supporting Growth of Osteoblast-like Cells. ACS Appl. Mater. Interfaces 2019, 11 (28), 24999-25007.

(43) Harvey, D.; Bardelang, P.; Goodacre, S. L.; Cockayne, A.; Thomas, N. R. Antibiotic Spider Silk: Site-Specific Functionalization of Recombinant Spider Silk Using "Click" Chemistry. Adv. Mater. 2017, 29 (10), 1604245. 